\title{
Using OCT Angiography as a Biomarker of Retinopathy Severity in Patients with Diabetes
}

\author{
Melanie Scheive, ${ }^{1}$ Kathryn Reinhart, ${ }^{1}$ Amir Hajrasouliha ${ }^{2,3}$ \\ ${ }^{1}$ Indiana University School of Medicine; ${ }^{2}$ Indiana University School of Medicine, Department of \\ Ophthalmology; ${ }^{3}$ Eugene and Marilyn Glick Eye Institute
}

Background and Hypothesis: Diabetic retinopathy (DR), the leading microvasculature and blindness-causing complication of diabetes mellitus, can be diagnosed and monitored using optical coherence tomography angiography (OCT-A) to visualize all retinal vasculature layers with greater resolution and limited invasiveness compared to the established technique of fluorescein angiography. We hypothesize that OCT-A can be used as a biomarker to correlate retinal vessel density (VD) from OCT-A with clinical severity of DR, visual acuity, and patient demographics as well as track anti-vascular endothelial growth factor (VEGF) treatment efficacy.

Methods: This retrospective cohort study analyzed the automatically quantified VDs of the superficial vascular complex (SVC) and deep vascular complex (DVC), including the whole, foveal, and parafoveal VDs, on quality OCT-A scans in patients with diagnosed DR. A multivariate linear regression and ANOVA analysis was completed to compare VDs to DR severity, visual acuity, and demographic factors in patients with at least one quality OCT-A scan. A linear mixed analysis was performed to determine how VD was affected by whether anti-VEGF therapy was given to patients with OCT-A scans at two or more different timepoints.

Results: There was found to be a positive correlation of the VDs in both the SVC whole and parafoveal VD and DVC parafoveal VD with decreased DR severity and increased visual acuity in the cross-sectional analysis ( $p \leq 0.001$ ). The DVC whole VD was also positively correlated with increased visual acuity $(p<0.001)$. There was no difference in the VDs associated with antiVEGF treatment over time.

Conclusions and Potential Impact: OCT-A shows promise for the diagnosis and monitoring of $\mathrm{DR}$ as a biomarker for disease severity which correlates with visual acuity. Anti-VEGF did not show significant change in VD in DR patients. Longer follow-up periods may be needed to elaborate on the long-term effects of anti-VEGF. 